\title{
The Structure and Meaning of Planck's Constant
}

\author{
David Humpherys \\ david.humpherys.wg00@wharton.upenn.edu
}

February 3, 2022

\begin{abstract}
Planck's constant plays an important role in quantum mechanics but has little physical meaning apart from its contribution to the formulas. Evaluating each of the constant's unit dimensions gives a more granular account of the physical phenomena described by the equations. These formulas show how classical and quantum mechanical equations are related, and they give consistent descriptions of phenomena like momentum and energy in classical and quantum systems.
\end{abstract}

Keywords: Planck constant, constant of proportionality, quantum of action, Planck length, Planck mass, Planck time

\section{Introduction}

Planck's constant plays a principal role in quantum physics, appearing regularly in formulas describing the natural world on small scales. The initial motivation for the constant was to explain the spectral radiance of black bodies, but its importance has grown over more than a century as the constant has been linked with a wide variety of quantum phenomena.

While Planck's constant plays an unambiguous role in the mathematical formulations of quantum theory, it is not immediately clear what physical attribute or dynamic it represents [1] or why it plays such an important role in nature. Its meaning is derived solely from the equations it appears in, yielding descriptions like the constant of proportionality and the quantum of action. In this regard, Planck's constant behaves like a function, transforming formula inputs into outputs while the function itself remains a mystery.

The key to deciphering the physical meaning of Planck's constant is in examining the transformations 
that take place in each of the constant's unit dimensions in the formulas and not simply calculating a result. A more granular view of the equations offers greater resolution and meaning.

\section{The Structure of Planck's constant}

Max Planck introduced the constant of proportionality $h$ as a fundamental constant of nature with a single value and unit dimensions $L^{2} M T^{-1}$. By combining the new constant with values and unit dimensions of the gravitational constant and speed of light, Planck derived quantities for each unit dimension shared by the three constants (see table 1 ).

Table 1: Max Planck derived relationships between the constants $\hbar, G$, and $c$ and natural units of length, mass, and time.

\begin{tabular}{ccc}
\hline Natural unit & Symbol & Planck formula \\
\hline \hline Planck length & $l_{P}$ & $\sqrt{\frac{\hbar G}{c^{3}}}$ \\
Planck mass & $m_{P}$ & $\sqrt{\frac{\hbar c}{G}}$ \\
Planck time & $t_{P}$ & $\sqrt{\frac{\hbar G}{c^{5}}}$ \\
\hline
\end{tabular}

These enigmatic ratios offer little or no ontological meaning for the concepts of length, mass, and time; yet the natural units are ingrained in formulas for calculating numerous physical phenomena.

An equivalent way of employing the universal constants is by expressing them in each unit dimension of length, mass, and time as shown in table 2. This approach has the considerable advantage of introducing more granular elements into the equations.

Table 2: Planck's constant, the gravitational constant, and the speed of light can be expressed as composite quantities of natural units. [2] [3] [4]

\begin{tabular}{rccc}
\hline Physical constant & Symbol & Natural unit formula & Simple formula \\
\hline \hline Speed of light & $c$ & $\frac{l_{P}}{t_{P}}$ & $c$ \\
Reduced Planck constant & $\hbar$ & $\frac{l_{P}^{2} m_{P}}{t_{P}}$ & $l_{P} m_{P} c$ \\
Gravitational constant & $G$ & $\frac{l_{P}^{3}}{m_{P} t_{P}^{2}}$ & $\frac{l_{P}}{m_{P}} c^{2}$ \\
\hline
\end{tabular}


That each of the universal constants may be neatly reduced to natural units of the correct magnitude and unit dimensions suggests that perhaps Planck should have presented the natural units as fundamental and the universal constants as their compound derivatives.

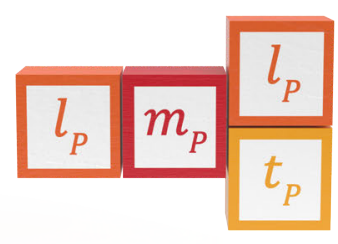

Figure 1: Planck's constant embodies natural units of length, mass, and time.

It is well-established that the speed of light is the ratio of Planck length to Planck time; and it is the concepts of length and time that give physical meaning to velocity. It is similarly the case that the physical meaning of Planck's constant is found in each of the unit dimensions it contributes to the formulas. These contributions are discussed in section 3 .

The question of whether to describe the physical universe in terms of Planck's constant and the gravitational constant, or in terms of the natural units they embody cannot be resolved mathematically. The formulas are equivalent and either approach produces the same result.

The case for deriving a better description of the physical world from natural units is made in this paper; first by demonstrating a mathematically equivalent form of the equations in natural units that contains more granular information; and second by showing that these formulas produce a consistent physical description of the universe that isn't apparent in the standard formulas.

A good starting point for understanding Planck's constant is challenging the assumption that $h$ and $G$ have meaning apart from the natural units they comprise. An important question is whether Planck's formulas in table 1 offer meaningful definitions of length, mass, and time. For example, does the speed of light to the fifth power represent something fundamental about time? Or is there another explanation for $c^{5}$ that suggests we shouldn't seek physical meaning from Planck's natural unit formulas?

One explanation for Planck's formulas is that they define elementary units of length, mass, and time using only compound quantities of those units. The reason that Planck's formula for time requires $c^{5}$ is because five units of $c$ are needed to eliminate all the quantities of length embedded in $\hbar$ and $G$. Only by removing units of length and mass can the formula produce the isolated quantity and unit dimension of time. A similar motive explains each of the formulas in table 3 . 
Table 3: Certain ratios of the compound constants are required to isolate individual unit dimensions of length, mass, and time.

\begin{tabular}{|c|c|c|}
\hline Natural unit & Planck formula & Natural unit formula \\
\hline Planck length & $\sqrt{\frac{\hbar G}{c^{3}}}$ & $\sqrt{\frac{l_{P}^{2} m_{P}}{t_{P}} \frac{l_{P}^{\prime}}{m_{P} t_{P}^{\prime}} \frac{t_{P}^{\prime}}{l_{P}^{\prime}}}=l_{P}$ \\
\hline Planck mass & $\sqrt{\frac{\hbar c}{G}}$ & $\sqrt{\frac{l_{P}^{\prime} m_{P}}{t_{P}} \frac{l_{P}}{t_{P}} \frac{m_{P} t_{P}^{\prime}}{l_{P}^{P}}}=m_{P}$ \\
\hline Planck time & $\sqrt{\frac{\hbar G}{c^{5}}}$ & $\sqrt{\frac{l_{P}^{f} m_{P}}{t_{P}} \frac{l_{P}^{\prime}}{m_{P} t_{P}^{\prime}} \frac{t_{P}^{2} t_{P}^{\prime}}{l_{P}^{\prime}}}=t_{P}$ \\
\hline
\end{tabular}

\section{Natural Unit Formulas}

The importance of natural units becomes clear when we replace Planck's constant with natural units of length, mass, and time in the formulas. One could imagine that these units would form no meaningful patterns if the constant is fundamental and the natural units are derivatives. But this is not the case. Instead, the natural units reveal a consistent pattern in the formulas by which physical attributes of a particle or system are proportional to their Planck scale limits. For example, the natural unit formula for momentum is written by replacing $\hbar$ with $l_{P} m_{P} c$ :

$$
p=\frac{\hbar}{\hbar}=\left(\frac{l_{P}}{\hbar}\right) m_{P} c
$$

The important structural insight is that Planck's constant supplies natural units of length and momentum. The formula input of wavelength in the denominator and the Planck length in the numerator produce a dimensionless ratio that quantifies a given particle's momentum proportional to the Planck momentum. Taken to its limit, we see that a photon with a Planck length wavelength has Planck momentum.

The natural unit formula suggests that it is not the composite value and dimensions of Planck's constant that define momentum. It is the ratio between Planck length and the particle's wavelength that quantifies the correct momentum.

Although one could say that momentum is proportional to both Planck's constant and the Planck momentum, a deeper examination of this pattern across formulas shows that it is generally the case that physical phenomena are proportional to their Planck scale limits whereas Planck's constant only provides certain Planck units which in this case are used for calculating momentum.

The Planck scale plays an important role in the equations of physics, giving a quantitative basis 


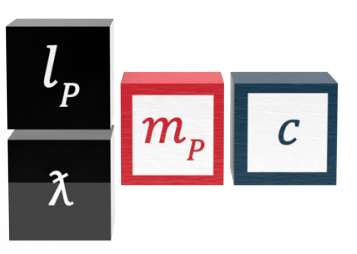

Figure 2: The natural momentum formula quantifies momentum in relation to the Planck momentum.

for calculating physical phenomena like momentum and energy at all scales. This is why we obtain meaningful results by setting certain constants like $\hbar, G$, and $c$ to one. However, the natural unit formulas offer a deeper level of insight and control over this natural structure. In the natural unit formulas, the role of each unit dimension is clarified whereas the composite constants are more difficult to manipulate. Most importantly, the natural unit formulas show that certain particle properties are correlated as shown in section 4

This natural pattern is not unique to photon momentum but repeats in formulas containing the universal constants [5]. In addition, natural formulas consistently describe mechanical, gravitational, and electromagnetic phenomena. Several examples of natural formulas are shown in appendix A] and any formula can be restated simply by replacing the composite constants with equivalent natural units.

The structure of natural unit formulas can be summarized in two parts as portrayed in figure 2

1. A Planck scale limit or basis in the unit dimensions of a physical phenomenon (mass, momentum, energy, force, etc.).

2. One or more dimensionless ratios between the physical phenomenon and the Planck scale.

In each formula, the conversion of universal constants into natural units produces the correct quantities in both parts of the function-natural units in the given unit dimensions, and dimensionless ratios in the correct proportions of the Planck scale.

The natural unit formula for photon energy is similar to the momentum formula, but because the photon's velocity is the speed of light, no additional inputs are required to quantify the photon's velocity. Photon energy is proportional to wavelength

$$
E=\frac{\hbar c}{\lambda}=\left(\frac{l_{P}}{\lambda}\right) E_{P}
$$


In the photon energy formula, Planck's constant and the speed of light produce Planck energy and Planck length. The photon's wavelength in the denominator makes the Planck length dimensionless and quantifies the photon's energy as a ratio of the Planck energy.

\subsection{Quantization}

Equations 1 and 2 show why Planck's constant is linked with the quantization of physical quantities like momentum and energy. Natural formulas clearly articulate two distinct levels of quantization that were described by Planck and Einstein in terms of Planck's constant [6] [7]. The quantization of energy for a single quantum of electromagnetic radiation is defined by equation 2 and was shown in section 3 to be limited to intervals of $l_{P} / \lambda$. The inverse wavelength ratio shows that intervals of a photon's energy become smaller as wavelength grows longer, asymptotically approaching zero energy. The range of possible energies is from a minimum limit of zero to the maximum Planck energy.

This natural energy scale shows that the possible energy states of a photon are not increments of a minimum unit in the same way that units of currency build up in multiples of a base unit. Photon energy can be arbitrarily small.

A second level of quantization is found in the separation of energy into individual packets of electromagnetic radiation which Einstein described in his seminal work on the photoelectric effect. The individual contributions of photons to radiant intensity is another level of quantization and one that more closely resembles the currency analogy.

These two levels of quantization give a structural description of the possible energy intervals represented by the natural formulas, but an additional consideration is the oscillator itself. The quantization of energy from a given set of oscillators may be further restricted by the states of those oscillators, as in the quantization of electron energies within the atom. [8].

\section{The structure of matter and electromagnetic radiation}

Classical and quantum physics are considered separate domains in which different rules and formulas apply. While it is generally believed that classical physics is the large-scale manifestation of quantum phenomena, the relationships between them-and the transition from one to the other-are not welldefined.

Natural formulas give a common language for describing both of these domains, showing explicitly 
how classical and quantum mechanical formulas are related. Writing the equations in natural units gives a single, coherent description of physical concepts like momentum, which today is characterized separately by two different formulas.

Humpherys has given a detailed description of this structure [2] which is briefly summarized here as it is essential for explaining the physical meaning of Planck's constant.

We can show that classical physics and quantum mechanics describe a common underlying structure by comparing the classical and quantum energy formulas. The classical kinetic energy formula

$$
E_{K}=\frac{1}{2} m_{0} v^{2}
$$

can be stated equivalently for a particle of matter such as an electron by expressing momentum and velocity as ratios of the Planck scale

$$
E_{K}=\frac{1}{2}\left(\frac{m_{0}}{m_{P}} \frac{v}{c}\right)\left(\frac{v}{c}\right) E_{P} .
$$

Note that the natural formula is also valid for a system of $n$ particles where the body's rest mass gives the correct extensive quantity of mass and velocity is an intensive property that works at any scale.

We can compare this structure with the quantum mechanical energy formula (2) in which the momentum and velocity components of the formula are each specified

$$
E_{\gamma}=\left(\frac{l_{P}}{\lambda}\right)\left(\frac{c}{c}\right) E_{P}
$$

Similarities are already evident in the structures of the classical and quantum formulas. But we can go further by resolving the apparent discord between the classical and quantum mechanical momentum formulas. Momentum is understood to pertain to both matter and radiation, but the different language of classical and quantum formulas conceals two important relationships. These correlations between the wavelength, momentum, and velocity of elementary particles are easily derived from the Compton and de Broglie wavelength formulas [2] and they are described in appendix $B$ for reference. These two relationships are summarized in table 4 
Table 4: Certain structural relationships of elementary particles are easily derived from the Compton and de Broglie wavelength formulas [2].

\begin{tabular}{cc}
$\beta_{\lambda}$ & $\beta_{v}$ \\
$\frac{l_{P}}{\lambda}=\frac{l_{P}}{\lambda_{C}} \frac{\lambda_{C}}{\lambda}=\frac{m_{0}}{m_{P}} \frac{v}{c}$ & $\frac{v}{c}=\frac{\lambda_{C}}{\lambda}$ \\
\hline
\end{tabular}

The symbol $\beta$ represents a ratio between 0 and 1 where 1 is the Planck scale. The subscript $\lambda$ designates the ratio of Planck length to wavelength and the subscript $v$ designates the ratio of velocity to the speed of light.

The essential insight is that rest mass and velocity are bulk properties of matter that give the same ratio of the Planck scale as particle wavelength. Rest mass quantifies a particle's Compton wavelength and velocity quantifies the ratio between its Compton wavelength and de Broglie wavelength. The Compton wavelength can be characterized more applicably here as the non-relativistic limit of the de Broglie wavelength as the particle's velocity approaches the speed of light.

Replacing classical momentum with the equivalent quantum form gives the following structural comparison between classical and quantum mechanical energy formulas

$$
\begin{aligned}
\frac{1}{2} m_{0} v^{2} & =\frac{1}{2}\left(\frac{l_{P}}{\lambda}\right)\left(\frac{v}{c}\right) E_{P} \\
\frac{\hbar c}{\lambda} & =1\left(\frac{l_{P}}{\lambda}\right)\left(\frac{c}{c}\right) E_{P}
\end{aligned}
$$

Table 5 demonstrates that the classical kinetic energy formula gives the same result as the natural unit formula, and the quantum formula is equal to the natural unit formula according to 2

Table 5: The classical kinetic energy formula is equivalent to the natural kinetic energy formula and shows how

\begin{tabular}{|c|c|c|c|c|c|}
\hline lepton & $\begin{array}{l}m_{0} \\
k g\end{array}$ & $\begin{array}{c}v \\
m / s\end{array}$ & $\begin{array}{l}\lambda \\
m\end{array}$ & $\begin{array}{c}E \\
\mathrm{kgm}^{2} / \mathrm{s}^{2}\end{array}$ & $\begin{array}{c}E \\
\mathrm{kgm}^{2} / \mathrm{s}^{2}\end{array}$ \\
\hline & CODATA & arbitrary & $\frac{\hbar}{p}=\left(\frac{m_{P}}{m_{0}}\right)\left(\frac{c}{v}\right) l_{P}$ & $1 / 2 m_{0} v^{2}$ & $\frac{1}{2}\left(\frac{l_{P}}{\lambda}\right)\left(\frac{v}{c}\right) E_{P}$ \\
\hline $\mathrm{e}$ & $9.109 \times 10^{-31}$ & $2,997,924$ & $3.862 \times 10^{-11}$ & $4.094 \times 10^{-18}$ & $4.094 \times 10^{-18}$ \\
\hline$\mu$ & $1.884 \times 10^{-28}$ & $1,000,000$ & $5.599 \times 10^{-13}$ & $9.418 \times 10^{-17}$ & $9.418 \times 10^{-17}$ \\
\hline$\tau$ & $3.168 \times 10^{-27}$ & $100,000,000$ & $3.329 \times 10^{-16}$ & $1.584 \times 10^{-11}$ & $1.584 \times 10^{-11}$ \\
\hline
\end{tabular}
classical and quantum formulas are related. 
The importance of wavelength and velocity ratios must be emphasized since they are embedded in both the classical and quantum formulas. Furthermore, the mechanical properties of elementary particles of matter and radiation can be quantified entirely from these two ratios as shown in table 6

Table 6: Classical and quantum formulas quantify the mechanical properties of matter and radiation using ratios of particle wavelength and velocity.

\begin{tabular}{rcccc}
\hline Physical property & Symbol & Ratios & Unit potential & Natural formula \\
\hline \hline Wavelength & $\lambda$ & $\frac{1}{\beta_{\lambda}}$ & $l_{P}$ & $\frac{l_{P}}{\beta_{\lambda}}$ \\
Rest mass & $m_{p}$ & $\frac{\beta_{\lambda}}{\beta_{v}}$ & $m_{P}$ & $\left(\frac{\beta_{\lambda}}{\beta_{v}}\right) m_{P}$ \\
Momentum & $p$ & $\beta_{\lambda}$ & $m_{P} c$ & $\beta_{\lambda} m_{P} c$ \\
Oscillation period & $T$ & $\frac{1}{\beta_{\lambda} \beta_{v}}$ & $t_{P}$ & $\frac{t_{P}}{\beta_{\lambda} \beta_{v}}$ \\
Kinetic energy & $E_{K}$ & $\beta_{\lambda} \beta_{v}$ & $E_{P}$ & $* \beta_{\lambda} \beta_{v} E_{P}$ \\
Total energy & $E$ & $\sqrt{\left(\frac{\beta_{\lambda}}{\beta_{v}}\right)^{2}+\beta_{\lambda}^{2}}$ & $E_{P}$ & $\sqrt{\left(\frac{\beta_{\lambda}}{\beta_{v}}\right)^{2}+\beta_{\lambda}^{2}} E_{P}$ \\
\hline
\end{tabular}

* Include a 1/2 coefficient for matter and 1 for radiation.

The natural formulas in table 6 consolidate classical and quantum mechanical formulas while simplifying the physical concepts. Various forms of classical and quantum formulas may be constructed simply by inserting the different forms of $\beta_{\lambda}$ and $\beta_{v}$ into the natural formulas in table 6. Some of the most common formulas are shown in table 7

\subsection{Two degrees of freedom}

The ratios $\beta_{\lambda}$ and $\beta_{v}$ quantify two degrees of freedom in the mechanical properties of elementary particles. Both matter and radiation have the same degree of freedom in their momenta, characterized physically by a particle's wavelength and not by velocity as the classical formula implies. Only the quantum mechanical description of momentum can be applied consistently to both matter and radiation. The ratio $l_{P} / \lambda$ correctly quantifies the momentum of both, even if $m_{0} v$ is the only practical method of measuring momentum in classical systems.

The second degree of freedom is a particle's velocity. Particles with rest mass have variability in their velocity while particles without rest mass do not. The correlation between a matter particle's wavelength 
Table 7: Natural unit formulas consolidate and explain classical and quantum mechanical formulas. Each formula in a given column produces the same result; each formula includes ratios of particle wavelength and velocity.

\begin{tabular}{|c|c|c|c|c|}
\hline $\begin{array}{c}\text { Wavelength } \\
m\end{array}$ & $\begin{array}{c}\text { Rest mass } \\
k g\end{array}$ & $\begin{array}{c}\text { Momentum } \\
\mathrm{kgm} / \mathrm{s}\end{array}$ & $\begin{array}{c}\text { Period } \\
s\end{array}$ & $\begin{array}{c}{ }^{*} \text { Kinetic energy } \\
\mathrm{kgm}^{2} / \mathrm{s}^{2}\end{array}$ \\
\hline \multirow[t]{2}{*}{$\frac{l_{P}}{\beta_{\lambda}}$} & $\frac{\beta_{\lambda}}{\beta_{v}} m_{P}$ & $\beta_{\lambda} m_{P} c$ & $\frac{t_{P}}{\beta_{\lambda} \beta_{v}}$ & $\beta_{\lambda} \beta_{v} E_{P}$ \\
\hline & & $\frac{l_{P}}{\lambda} m_{P} c$ & $\frac{\lambda}{l_{P}} \frac{c}{v} t_{P}$ & $\frac{l_{P}}{\lambda} \frac{v}{c} E_{P}$ \\
\hline$\hbar$ & & $\hbar$ & $\lambda$ & $\hbar c$ \\
\hline \multirow[t]{2}{*}{$\bar{p}$} & & $\bar{\lambda}$ & $\bar{v}$ & $\bar{t}$ \\
\hline & & & $\frac{E_{P}}{E} t_{P}$ & $\frac{t_{P}}{T} E_{P}$ \\
\hline$\frac{m_{P}}{m_{0}} \frac{c}{v} l_{P}$ & $\frac{l_{P}}{\lambda_{C}} m_{P}$ & $\frac{l_{P}}{\lambda_{C}} m_{P} \frac{\lambda_{C}}{\lambda} c$ & & $\frac{1}{2} \frac{l_{P}}{\lambda_{C}} \frac{\lambda_{C}}{\lambda} \frac{v}{c} E_{P}$ \\
\hline$\frac{\hbar}{m_{0} v}$ & & $m_{0} v$ & & $\frac{1}{2} m_{0} v^{2}$ \\
\hline
\end{tabular}

* Kinetic energy formulas require a $1 / 2$ coefficient for matter and 1 for radiation. Energy formulas in the table that are stated in terms of rest mass display the one-half coefficient.

and velocity suggests an equipartition of energy across the two degrees of freedom. This correlation can be expressed in three statements about changes in the wavelength, momentum, and velocity of matter particles

$$
\Delta \lambda \Delta v=1
$$

$$
\Delta \lambda \Delta p=1
$$

$$
\frac{\Delta p}{\Delta v}=1
$$

For matter and radiation, kinetic energy is characterized by two components:

1. An energy potential generated by a particle's wavelength, proportional to $\frac{l_{P}}{\lambda}$.

2. The particle's velocity.

The product of these two degrees of freedom accurately quantifies the kinetic energy of matter and radiation 1 as shown in equations 6 and 7 , and table 5

\footnotetext{
${ }^{1}$ a $1 / 2$ coefficient is also required for matter.
} 


\subsection{Inertial mass}

The conflicting descriptions of momentum given by classical and quantum mechanical momentum formulas can be resolved by treating momentum as a function of wavelength instead of rest mass and velocity. Removing unit dimensions of velocity from momentum gives a quantity of inertial mass $m$ that is inversely proportional to a particle's wavelength

$$
\frac{l_{P}}{\lambda}=\frac{m}{m_{P}} .
$$

Inertial mass should not be confused with rest mass. The natural formulas describe the effect of rest mass as reducing a massive particle's energy potential from the Planck scale down to the Compton wavelength scale, where the Compton wavelength represents the minimum limit of the particle's wavelength at the velocity limit, $c$. The particle's inertial mass is less than its rest mass by the ratio $v / c$

$$
m=m_{0} \frac{v}{c}=\frac{p}{c}
$$

and can easily be calculated by dividing any quantity of momentum by $c$.

The relationship between inertial mass and rest mass is also described by the energy-momentum relation. Replacing momentum with inertial mass in the formula gives a vector sum of the two masses

$$
E=\sqrt{m^{2}+m_{0}^{2}} c^{2} .
$$

\subsection{The physical structure of kinetic energy}

The structure of matter and radiation described by natural unit formulas is shown in figure 3 The figure compares the kinetic energies of a photon and an electron; the wavelength degree of freedom is shown in orange and the velocity degree of freedom in blue. Each of the particles has the same wavelength while the photon's velocity is $c$ and the electron's velocity is $v$.

As the illustration shows, the inertial mass of the photon and electron are equal since the particles have the same wavelength. Multiplying inertial mass by velocity gives the kinetic energy of each particle; but note that each result must be multiplied by a superfluous quantity of $c$ to obtain the familiar unit dimensions of energy, and matter requires a one-half coefficient. 


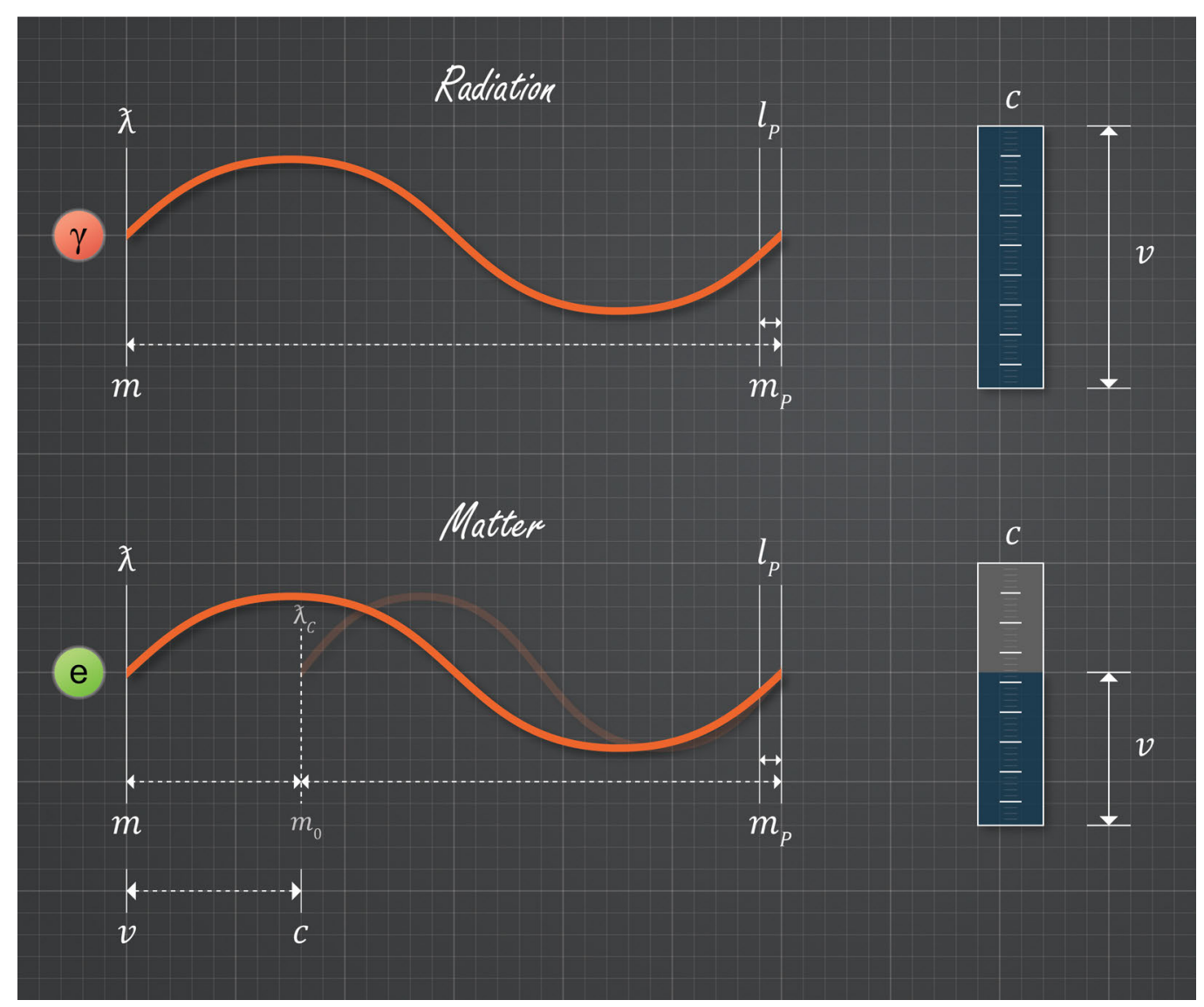

Figure 3: The kinetic energy of matter and radiation can be characterized physically as the product of two factors-an energy potential inversely proportional to wavelength and the particle's velocity.

One might ask the question, since $m c=m_{0} v$ and either formula produces the same result, what is the purpose of restating momentum as inertial mass? The answer is simply that while the calculated results are the same, the physical states represented by the two formulas are different. One state describes momentum as mass in motion, and attributes changes in momentum to changes in velocity. This state can only be applied to matter.

The second state characterizes momentum as a kind of mass density or energy potential that is inversely proportional to a particle's wavelength. This state can be applied equally to matter and radiation.

A worthy standard for encoding physical quantities as mathematical symbols and operations is the proper alignment between the symbols and operations in the formula and the physical attributes and dynamics they represent. This standard demands greater contextual awareness of a formula's physical meaning in a language besides mathematics. Without this accountability, formulas become their own ontologies no matter how they are expressed.

For example, the physical characterization of kinetic energy as a function of particle wavelength and velocity explains why the kinetic energy of matter is proportional to velocity squared. Since a change in 
velocity is accompanied by a proportional change in wavelength according to 33 only one instance of velocity characterizes kinetic energy. The second instance of $v$ is the proportional relationship between velocity and wavelength.

Figure 4 illustrates how the wavelength and velocity description of kinetic energy produces the correct quantitative result while also giving a meaningful and consistent physical description of kinetic energy.

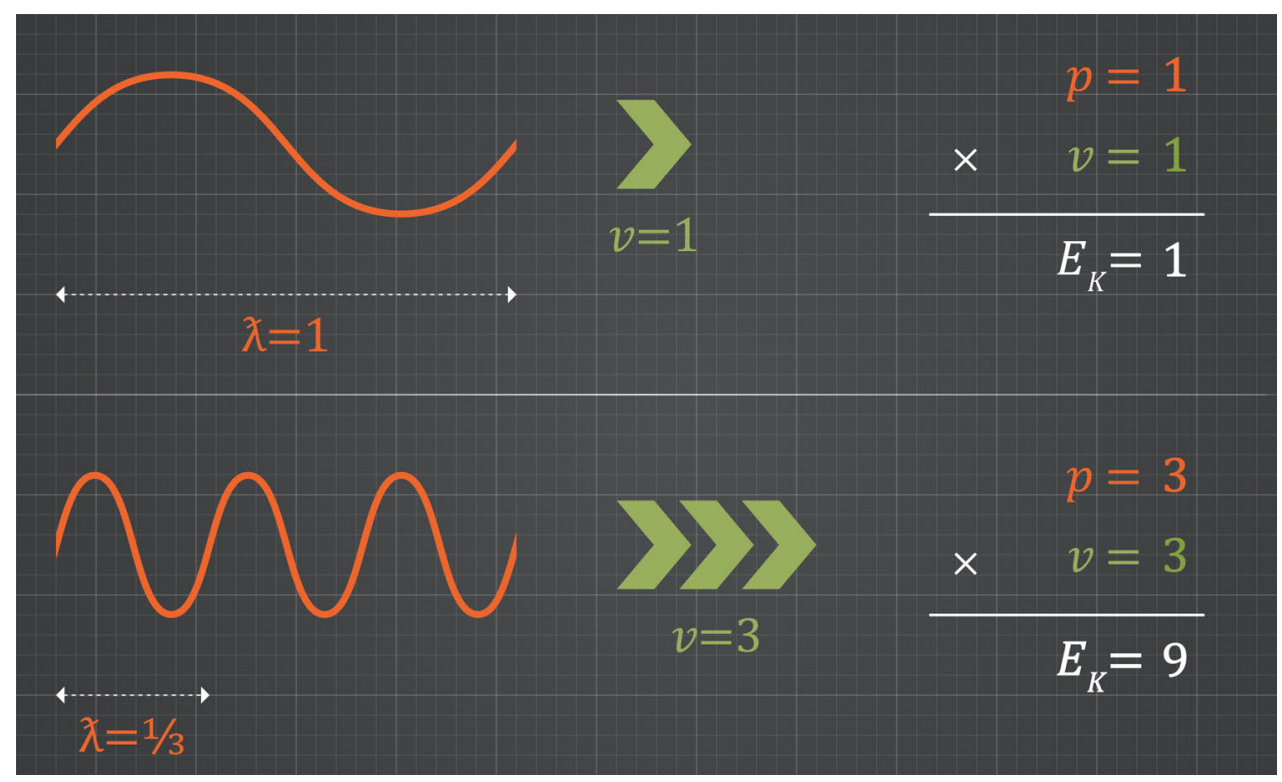

Figure 4: The two-part structure of energy explains why the kinetic energy of matter is proportional to velocity squared. Energy is spread equally across a matter particle's wavelength and velocity degrees of freedom, correlating these two physical properties.

\section{$4.4 E=m_{0} c^{2}$}

The simple two-part structure of energy described by natural unit formulas and illustrated in figure 3 gives a physical explanation of mass-energy equivalence. To see how, consider three equivalent energy formulas expressed in classical, quantum mechanical, and natural terms

$$
E=m_{0} c^{2}=\frac{\hbar c}{\lambda_{C}}=\left(\frac{l_{P}}{\lambda_{C}}\right) E_{P} .
$$

The physical description of 14 is that a matter particle's latent rest mass has a kinetic energy potential equal to the radiant energy of a photon with the particle's Compton wavelength. Equation 13 shows how this rest mass component combines with the particle's inertial mass component, quantified by its wavelength, to formulate the total energy. 
The structure illustrated in figures 3 and 4 also gives a meaningful physical interpretation of the ratio

$$
\frac{v^{2}}{c^{2}}
$$

which appears in the Lorentz transformation governing the behavior of relativistic interactions. Since one of the $v$ terms is proportional to changes in wavelength, the combined ratio $v^{2} / c^{2}$ quantifies the combined difference between a particle's wavelength and its minimum wavelength limit; and between its velocity and the speed of light.

\section{Quantum of action}

An examination of the mechanical properties of charged leptons and photons shows why Planck's constant is so important for calculating particle dynamics. In addition to representing natural units of length, mass, and time, Planck's constant is also a conserved quantity in certain combinations of elementary particle properties at any scale. The following pairs of properties are conserved as shown in table 8

$$
\begin{gathered}
\lambda m=l_{P} m_{P}=3.518 \times 10^{-43} \mathrm{kgm} \\
\lambda p=\lambda m c=l_{P} m_{P} c=1.055 \times 10^{-34} \mathrm{kgm}^{2} / \mathrm{s} \\
T E=t_{P} E_{P}=1.055 \times 10^{-34} \mathrm{kgm}^{2} / \mathrm{s} .
\end{gathered}
$$

Equations 17 and 18 have unit dimensions of action, and because the combinations of these properties for an elementary particle are equal to Planck's constant, the constant is sometimes called the quantum of action.

Each of the conserved pairs in 16,17 and 18 may be considered a symmetry in which the product of the pair is invariant to changes in either term. Furthermore, any pair is equivalent to the Planck unit values of the pair. Although they appear as three different symmetries, they are all reflections of a single symmetry between particle wavelength and inertial mass. Figure 5 illustrates why this is the case. 
Table 8: Certain pairs of particle properties are conserved including wavelength-inertial mass, wavelengthmomentum, and time-energy. All three relationships can be explained in terms of a single length-mass symmetry.

\begin{tabular}{|c|c|c|c|c|c|c|c|}
\hline & $\lambda_{C}$ & $m_{0}$ & $\lambda_{C} m_{0}$ & $v$ & $\lambda$ & $m$ & $\lambda m$ \\
\hline & $m$ & $\mathrm{~kg}$ & kgm & $\mathrm{m} / \mathrm{s}$ & $m$ & $\mathrm{~kg}$ & $\mathrm{kgm}$ \\
\hline & CODATA & CODATA & - & *arbitrary & $\frac{l_{P}}{\beta_{\lambda}}$ & $\beta_{\lambda} m_{P}$ & - \\
\hline e & $3.86 \times 10^{-13}$ & $9.11 \times 10^{-31}$ & $3.52 \times 10^{-43}$ & *2, 997,924 & $3.86 \times 10^{-11}$ & $9.11 \times 10^{-33}$ & $3.52 \times 10^{-43}$ \\
\hline$\mu$ & $1.87 \times 10^{-15}$ & $1.88 \times 10^{-28}$ & $3.52 \times 10^{-43}$ & ${ }^{*} 1,000,000$ & $5.60 \times 10^{-13}$ & $6.28 \times 10^{-31}$ & $3.52 \times 10^{-43}$ \\
\hline$\tau$ & $1.11 \times 10^{-16}$ & $3.17 \times 10^{-27}$ & $3.52 \times 10^{-43}$ & ${ }^{*} 100,000,000$ & $3.33 \times 10^{-16}$ & $1.06 \times 10^{-27}$ & $3.52 \times 10^{-43}$ \\
\hline \multirow[t]{4}{*}{$\gamma$} & - & - & - & $299,792,458$ & ${ }^{*} 2.00 \times 10^{-8}$ & $1.76 \times 10^{-35}$ & $3.52 \times 10^{-43}$ \\
\hline & $p$ & $T$ & $E$ & $\lambda p$ & ${ }^{\S} T E$ & & \\
\hline & $\mathrm{kgm} / \mathrm{s}$ & $s$ & $\mathrm{kgm}^{2} / \mathrm{s}^{2}$ & $\mathrm{kgm}^{2} / \mathrm{s}$ & $\mathrm{kgm}^{2} / \mathrm{s}$ & & \\
\hline & $\beta_{\lambda} m_{P} c$ & $\frac{t_{P}}{\beta_{\lambda} \beta_{v}}$ & ${ }^{\dagger} \beta_{\lambda} \beta_{v} E_{P}$ & - & - & & \\
\hline e & $2.73 \times 10^{-24}$ & $1.29 \times 10^{-17}$ & $4.09 \times 10^{-18}$ & $1.05 \times 10^{-34}$ & $1.05 \times 10^{-34}$ & & \\
\hline$\mu$ & $1.88 \times 10^{-22}$ & $5.60 \times 10^{-19}$ & $9.42 \times 10^{-17}$ & $1.05 \times 10^{-34}$ & $1.05 \times 10^{-34}$ & & \\
\hline$\tau$ & $3.17 \times 10^{-19}$ & $3.33 \times 10^{-24}$ & $1.58 \times 10^{-11}$ & $1.05 \times 10^{-34}$ & $1.05 \times 10^{-34}$ & & \\
\hline$\gamma$ & $5.27 \times 10^{-27}$ & $6.67 \times 10^{-17}$ & $1.58 \times 10^{-18}$ & $1.05 \times 10^{-34}$ & $1.05 \times 10^{-34}$ & & \\
\hline
\end{tabular}

Velocities of the charged leptons were chosen arbitrarily and any velocity gives similar results. The photon's wavelength was also chosen arbitrarily and any wavelength gives similar results.

$\S \times 2$ for matter and 1 for radiation

$\dagger \times 0.5$ for matter and 1 for radiation

The combination $\lambda m$ is the physical representation of all three symmetries. A given wavelength has an inversely proportional quantity of inertial mass, and this is true for matter and radiation.

The pairs $\lambda p$ and $T E$ are conserved because each of them multiplies $t m$ by constants. Wavelengthmomentum multiplies the symmetrical pair $\lambda m$ by the speed of light, and Time-energy multiplies that by the particle's oscillation period in the numerator and denominator.

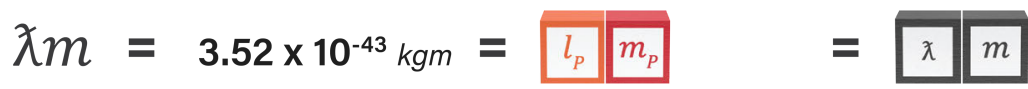

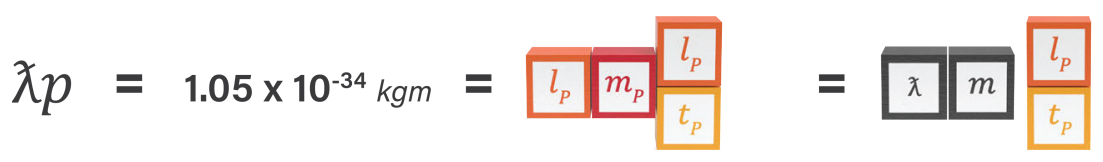

$$
\begin{aligned}
& T E=1.05 \times 10^{-34} \mathrm{kgm}=\begin{array}{|l|l|l|l|l|l|l|}
\hline l_{P} & m_{P} & l_{P} & t_{P} \\
\hline t_{P} & t_{P}
\end{array}=\begin{array}{ll|l|l|l|}
\hline & l_{P} & T \\
\hline
\end{array}
\end{aligned}
$$

Figure 5: All three conserved pairs are reflections of a single symmetry between wavelength and inertial mass. 
These symmetries and the structure of matter and radiation demonstrated and explained in section 4 show that Planck's constant includes a physically unrepresentative quantity of $c$ because formulas are stated in terms of velocity rather than wavelength. A more representative expression of the constant is the product of Planck length and Planck mass, $l_{P} m_{P}=\frac{\hbar}{c}=3.51767294 \times 10^{-43} \mathrm{kgm}$.

\section{Quantum spin}

Planck's constant is linked with the quantum phenomenon of angular momentum. Bohr first proposed that electron angular momentum is quantized in units of Planck's constant [8]

$$
L=m_{0} v r=n \hbar
$$

The subsequent application of the Schrodinger equation to the atomic model incorporated the same idea.

Bohr had observed discrete electron orbitals and assigned integer numbers to them. It is easy to see from 19 how one could presume that Planck's constant is a discrete unit of angular momentum that is responsible for creating the orbital intervals. But while $n \hbar$ quantifies the correct electron wavelengths in each orbital, the meaning of Planck's constant in the formula is more subtle. It may be that something else is responsible for quantizing the orbitals and Planck's constant is simply used to calculate electron properties.

Restating equation 19 as a ratio between radius and wavelength gives the de Broglie wavelength formula

$$
\frac{r_{n}}{n}=\frac{\hbar}{m_{0} v}=\frac{m_{P}}{m_{0}} \frac{c}{v} l_{P}
$$

which is not generally limited to atomic orbitals. Bohr published his paper more than a decade before Louis de Broglie identified the equation as a general formula for calculating the wavelengths of matter particles. Furthermore, the role of Planck's constant in the formula is to inject natural units and does not resemble a discrete unit that grows in multiples of atomic orbitals. The association of Planck's constant with quantum spin is likely because Planck's constant is conserved over any integer wavelength as demonstrated in section 5

Formulas show that the quantization of atomic orbitals may be attributed to the fine-structure constant 
rather than Planck's constant. The Rydberg energy formula [9]

$$
h c R_{\infty}=\frac{1}{2} m_{e} \alpha^{2} c^{2}
$$

quantifies the electron ground state energy using the classical kinetic energy formula. Given the physical structure of kinetic energy shown in section 4 the fine-structure constant can be interpreted as quantifying equivalent ratios of wavelength

$$
\alpha=\frac{\lambda_{C}}{\lambda}
$$

and velocity

$$
\alpha=\frac{v}{c} .
$$

in the ground state.

The electron wavelength in the $n t h$ orbital can be stated in multiples of the ground state wavelength and therefore in terms of the fine-structure constant

$$
\lambda_{n}=n\left(\frac{\lambda_{C}}{\alpha}\right)
$$

\section{Heisenberg uncertainty relation}

Perhaps the most recognized use of Planck's constant is in the Heisenberg uncertainty relation. In this context, the constant tells us something about the limits in which certain physical quantities can be specified simultaneously.

The Heisenberg uncertainty relation is stated as an inequality but its structure closely resembles equalities demonstrated throughout this paper in more granular terms and unit dimensions. An examination of the uncertainty relation should first consider these equalities including the structure of matter described in section 4 In particular, the relationships between wavelength, mass, and velocity shown in table 4 and section $B$ are important, as well as equations $8-\sqrt{10}$ and $16-18$, These structural relationships are also summarized in figure 3 .

The Heisenberg uncertainty relation offers more information when Planck's constant is stated in 
natural units. The common form of the relation

$$
\Delta x \Delta p \geq \frac{\hbar}{2}
$$

can be expanded into individual terms and unit dimensions

$$
\Delta x m_{0} \Delta v \geq \frac{1}{2} l_{P} m_{P} c
$$

As with other natural formulas, it is the ratios between physical attributes and the Planck scale that quantify a physical system. The formula compares ratios of position/length, rest mass, and velocity with Planck scale quantities of length, mass, and time. Arranging the formula in terms of the uncertainty in position $x$ gives

$$
\Delta x \geq \frac{1}{2}\left(\frac{m_{P}}{m_{0}}\right)\left(\frac{c}{\Delta v}\right) l_{P} .
$$

In 27 the variable components of the inequality are position and velocity. This much is clearly understood, but the physical structure generally is not. The two variables are related by 8 and 33 which show that changes in matter particle wavelength are proportional to velocity. The ratio $m_{P} / m_{0}$ multiplied by the Planck length quantifies the fixed length of the particle's Compton wavelength-the minimum limit of its physical wavelength. The ratio $c / v$ re-quantifies this length to the full de Broglie wavelength at the given velocity $v$, according to 33 . Equation 27 can be stated explicitly in terms of wavelength by substituting the intensive ratio $c / v$ with the same ratio $\lambda / \lambda_{C}$ (note that this removes unit dimensions of velocity from the formulas)

$$
\Delta x \geq \frac{1}{2}\left(\frac{\lambda_{C}}{l_{P}}\right)\left(\frac{\Delta \lambda}{\lambda_{C}}\right) l_{P} .
$$

The formulas support the conclusion that the Heisenberg uncertainty relation is a statement about the incompatibility of simultaneously characterizing matter as both a particle and a wave. However, the uncertainty is not over the full particle wavelength but only half of it. A possible explanation is that only the orthogonal directions of a particle's phase are positively distinguishable by any single measurement.

An alternative form of the uncertainty relation

$$
\Delta T \Delta E \geq \frac{\hbar}{2}
$$

can be explained in the same terms as shown in section 5 The time-energy relation multiplies the 
wavelength-momentum relation by the constant $T / T$

$$
\Delta x \geq \frac{1}{2}\left(\frac{\lambda_{C}}{l_{P}}\right)\left(\frac{\Delta \lambda}{\lambda_{C}}\right)\left(\frac{T}{T}\right) l_{P} .
$$

\section{Conclusion}

More than a century after Max Planck introduced the constant of proportionality, the ontological meaning of $h$ is still unclear. Equations with Planck's constant are black box functions that transform formula inputs into outputs but the function remains a mystery.

Deconstructing the composite value and unit dimensions of Planck's constant into distinct units of length, mass, and time transforms black box functions into meaningful descriptions of physical phenomena. These formulas show how classical and quantum formulas are different but equivalent ways of representing a single, common structure underlying the mechanics of elementary particles of matter and radiation.

According to natural unit formulas, Planck's constant is not a discrete unit or a single physical quantity, concept, or idea, but a compound mixture of values and dimensions that can be understood in two ways:

1. A versatile set of length, mass, and time units, $\frac{l_{P}^{2} m_{P}}{t_{P}}$, that is useful for quantifying certain observable physical phenomena in proportion to the Planck scale.

2. A conserved quantity of certain elementary particle properties including a particle's wavelengthmomentum and time-energy. These conservations are reflections of a single, fundamental symmetry between wavelength and inertial mass.

Natural unit formulas challenge various assumptions about the boundaries between quantum and classical physics, as well as the extent of mathematical abstraction needed to characterize the quantum world. Natural unit formulas give a physically meaningful description of Planck's constant and natural phenomena like momentum and energy on quantum and classical scales. 


\section{A Natural formulas}

The following illustration gives examples of how formula inputs combine with Planck units to produce natural unit formulas. These formulas show that observable physical phenomena are quantified in proportion to the Planck scale.

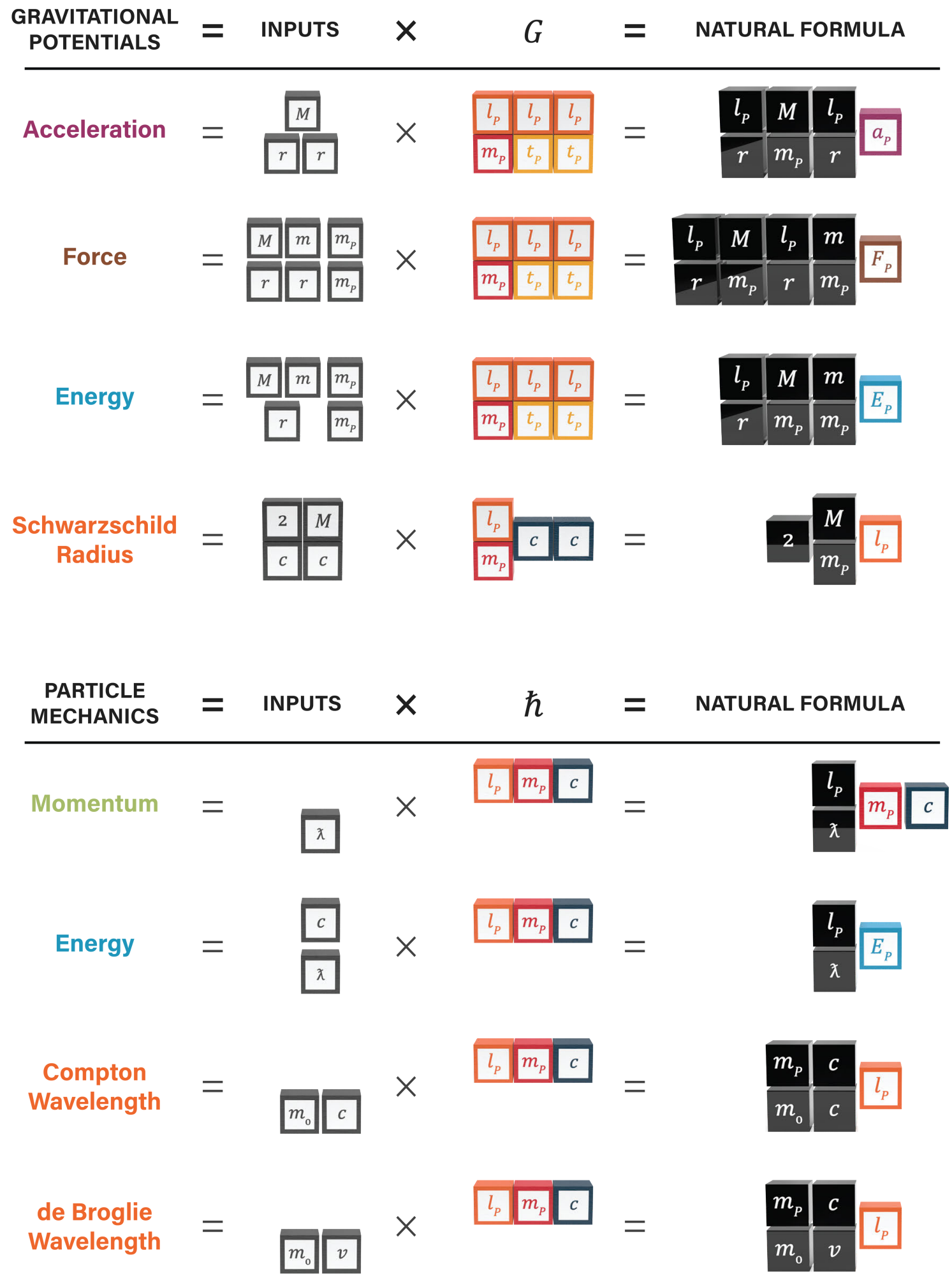




\section{B Structural relationships of elementary particles}

Several important structural relationships of matter and radiation are represented in table 7 . These relationships are explained by Humpherys [2] and summarized below.

\section{B.1 Correlation between Compton wavelength and rest mass}

Rest mass is inversely proportional to Compton wavelength [10], [11], [12]. The product of rest mass and Compton wavelength is a constant, equal to the product of Planck length and Planck mass

$$
\lambda_{C} m_{0}=l_{P} m_{P}=3.518 \times 10^{-43} \mathrm{kgm}
$$

This relationship can also be expressed as an equivalence between dimensionless ratios of wavelength and mass.

$$
\frac{l_{P}}{\lambda_{C}}=\frac{m_{0}}{m_{P}}
$$

The following table demonstrates the relationship between rest masses and Compton wavelengths

\begin{tabular}{|c|c|c|c|c|c|c|}
\hline Lepton & $\begin{array}{r}\lambda_{C} \\
m\end{array}$ & $\begin{array}{l}m_{0} \\
k g\end{array}$ & $\begin{array}{c}\lambda_{C} m_{0} \\
k g m\end{array}$ & $\begin{array}{c}l_{P} m_{P} \\
k g m\end{array}$ & $\frac{l_{P}}{\lambda_{C}}$ & $\begin{array}{c}\frac{m_{0}}{m_{P}} \\
-\end{array}$ \\
\hline e & $3.862 \times 10^{-13}$ & $9.109 \times 10^{-31}$ & $3.518 \times 10^{-43}$ & $3.518 \times 10^{-43}$ & $4.185 \times 10^{-23}$ & $4.185 \times 10^{-23}$ \\
\hline$\mu$ & $1.868 \times 10^{-15}$ & $1.884 \times 10^{-28}$ & $3.518 \times 10^{-43}$ & $3.518 \times 10^{-43}$ & $8.654 \times 10^{-21}$ & $8.654 \times 10^{-21}$ \\
\hline$\tau$ & $1.111 \times 10^{-16}$ & $3.168 \times 10^{-27}$ & $3.518 \times 10^{-43}$ & $3.518 \times 10^{-43}$ & $1.455 \times 10^{-19}$ & $1.455 \times 10^{-19}$ \\
\hline
\end{tabular}
of the charged leptons.

\section{B.2 Correlation between wavelength and velocity}

Kinetic energy is spread equally across wavelength and velocity degrees of freedom in a matter particle. This equipartition of energy gives a relationship between the particle's wavelength and velocity

$$
\frac{\lambda_{C}}{\lambda}=\frac{v}{c}
$$

The following table demonstrates the relationship between wavelength and velocity. Velocities in the table are arbitrary and any velocity will give similar results. 


\begin{tabular}{ccccccc}
\hline \multirow{2}{*}{ Lepton } & $\lambda_{C}$ & $m_{0}$ & $v$ & $\hbar$ & $\frac{\lambda_{C}}{\hbar}$ & $\frac{v}{c}$ \\
& $m$ & $k g$ & $m / s$ & $m$ & - & - \\
\hline $\mathrm{e}$ & $3.862 \times 10^{-13}$ & $9.109 \times 10^{-31}$ & $2,997,924$ & $3.862 \times 10^{-11}$ & $\mathbf{0 . 0 1 0 0}$ & $\mathbf{0 . 0 1 0 0}$ \\
$\mu$ & $1.868 \times 10^{-15}$ & $1.884 \times 10^{-28}$ & $1,000,000$ & $5.599 \times 10^{-13}$ & $\mathbf{0 . 0 0 3 3}$ & $\mathbf{0 . 0 0 3 3}$ \\
$\tau$ & $1.111 \times 10^{-16}$ & $3.168 \times 10^{-27}$ & $100,000,000$ & $3.329 \times 10^{-16}$ & $\mathbf{0 . 3 3 3 6}$ & $\mathbf{0 . 3 3 3 6}$ \\
\hline
\end{tabular}

\section{B.3 Correlation between $m_{0} v$ and $t$}

The combination of 32 and 33 show how classical and quantum mechanical momentum formulas are equivalent. Rest mass quantifies a particle's Compton wavelength and velocity quantifies the ratio between the Compton wavelength and de Broglie wavelength.

$$
\frac{l_{P}}{\lambda}=\frac{m_{0}}{m_{P}} \frac{v}{c}=\frac{l_{P}}{\lambda_{C}} \frac{\lambda_{C}}{\hbar}
$$

The following table demonstrates the relationship between wavelength and velocity. Velocities shown are arbitrary and the non-relativistic relationship holds for all velocities.

\begin{tabular}{ccccccc}
\hline \multirow{2}{*}{ Lepton } & $\lambda_{C}$ & $m_{0}$ & $v$ & $\lambda$ & $\frac{l_{P}}{\hbar}$ & $\frac{m_{0} v}{m_{P} c}$ \\
& $m$ & $k g$ & $m / s$ & $m$ & - & - \\
\hline $\mathrm{e}$ & $3.862 \times 10^{-13}$ & $9.109 \times 10^{-31}$ & $2,997,924$ & $3.862 \times 10^{-11}$ & $\mathbf{4 . 1 8 5} \times \mathbf{1 0}^{-25}$ & $\mathbf{4 . 1 8 5} \times \mathbf{1 0}^{-\mathbf{2 5}}$ \\
$\mu$ & $1.868 \times 10^{-15}$ & $1.884 \times 10^{-28}$ & $1,000,000$ & $5.599 \times 10^{-13}$ & $\mathbf{2 . 8 8 7} \times \mathbf{1 0}^{-23}$ & $\mathbf{2 . 8 8 7} \times \mathbf{1 0}^{-23}$ \\
$\tau$ & $1.111 \times 10^{-16}$ & $3.168 \times 10^{-27}$ & $100,000,000$ & $3.329 \times 10^{-16}$ & $\mathbf{4 . 8 5 5} \times \mathbf{1 0}^{-\mathbf{2 0}}$ & $\mathbf{4 . 8 5 5} \times \mathbf{1 0}^{-20}$ \\
\hline
\end{tabular}

The following table demonstrates that momentum is a function of wavelength for matter and radiation, regardless of whether a particle has rest mass or whether its velocity varies. The electron velocity shown was chosen arbitrarily and the muon and tau velocities were calculated to give the same wavelength as the electron. The photon wavelength was selected to match the lepton wavelengths.

\begin{tabular}{|c|c|c|c|c|}
\hline \multirow[t]{2}{*}{ Particle } & $m_{0}$ & $v$ & $\lambda$ & $p$ \\
\hline & $\mathrm{kg}$ & $\mathrm{m} / \mathrm{s}$ & $m$ & $\mathrm{kgm} / \mathrm{s}$ \\
\hline e & $9.109 \times 10^{-31}$ & $2,997,924$ & $3.862 \times 10^{-11}$ & $2.731 \times 10^{-24}$ \\
\hline$\mu$ & $1.884 \times 10^{-28}$ & 14,499 & $3.862 \times 10^{-11}$ & $2.731 \times 10^{-24}$ \\
\hline$\tau$ & $3.168 \times 10^{-27}$ & 862 & $3.862 \times 10^{-11}$ & $2.731 \times 10^{-24}$ \\
\hline$\gamma$ & - & $299,792,458$ & $3.862 \times 10^{-11}$ & $2.731 \times 10^{-24}$ \\
\hline
\end{tabular}




\section{References}

[1] D. C. Chang, "Physical interpretation of Planck's constant based on the maxwell theory," Chinese Physics B, vol. 26, no. 4, p. 040301, 2017.

[2] D. Humpherys, "Natural Planck Units and the Structure of Matter and Radiation," Quantum Speculations, vol. 3, 2021.

[3] T. Roberts, Einstein's Intuition: Visualizing Nature in Eleven Dimensions. Quantum Space Theory Institute, 2016.

[4] J. A. Geiger, "Measurement Quantization Describes History of Universe-Quantum Inflation, Transition to Expansion, CMB Power Spectrum," Journal of High Energy Physics, Gravitation and Cosmology, vol. 6, no. 2, pp. 186-224, 2020.

[5] D. Humpherys, "On the Fundamental Constants of Nature," Preprints, 2020.

[6] M. Planck, “Über irreversible Strahlungsvorgänge," Sitzungsberichte der Königlich Preußischen Akademie der Wissenschaften zu Berlin, vol. 5, no. 1, pp. 440-480, 1899.

[7] A. Einstein, “Über einem die Erzeugung und Verwandlung des Lichtes betreffenden heuristischen Gesichtspunkt," Annalen der physik, vol. 4, 1905.

[8] N. Bohr, "I. On the Constitution of Atoms and Molecules," The London, Edinburgh, and Dublin Philosophical Magazine and Journal of Science, vol. 26, no. 151, pp. 1-25, 1913.

[9] "Fundamental Physical Constants," https://physics.nist.gov/cuu/Constants/index.html, 2022.

[10] G. R. Kepner, "Relating the debroglie and compton wavelengths to the velocity of light?," Applied Physics Research, vol. 10, no. 4, 2018.

[11] E. G. Haug, "Collision-space-time: Unified quantum gravity," Physics Essays, vol. 33, no. 1, pp. 46$78,2020$.

[12] E. G. Haug, "Newton's and Einstein's Gravity in a New Perspective for Planck Masses and Smaller Sized Objects," International Journal of Astronomy and Astrophysics, vol. 8, no. 1, pp. 6-23, 2018. 